\title{
Verbesserung des Recyclings von Elektrokleingeräten bei Erstbehandlern und Steigerung der Metallausbeute durch Einsatz des Magnetabscheiders
}

\author{
Christian Borowski, Andreas Glimm und Michael Pieplow \\ Hochschule Nordhausen, Nordhausen, Deutschland
}

Eingegangen 16. Januar 2021; angenommen 1. Februar 2021; online publiziert 23. Februar 2021

\begin{abstract}
Zusammenfassung: In den vergangenen Jahren hat sich die Nutzung von Wertstoffen aus Elektroaltgeräten auf Grund von Quotenregelungen erhöht. Die Verbesserung der Ausbringung von Wertstoffen ist ein Ziel, mit dem sich die hier beschriebenen Forschungsvorhaben beschäftigen. Untersucht wurden Möglichkeiten zur verbesserten Separierung von Metallen aus Elektrokleingeräten bei Erstbehandlern durch den Einsatz marktverfügbarer und kostengünstiger Aufbereitungstechnik und Optimierungspotentiale unter Verwendung eines entwickelten Multimetallseparators. Auftretende Probleme sowie Aspekte einer repräsentativen Beprobung von zerkleinertem Elektroschrott $<8 \mathrm{~mm}$ wurden ebenso betrachtet.
\end{abstract}

Schlüsselwörter: Wertstoffrückgewinnung, Elektroaltgeräte, Haushaltskleingeräte, Elektroschrott, Erstbehandler, Magnet, Separation, Feinkorn, Multimetallseparator, Probenahme

Improving the Recycling of Small Electrical Equipment in the First Treatment and Increasing the Metal Yield by Using the Magnetic Separator

Abstract: In recent years, the use of recyclable materials from old electrical appliances has increased due to quota regulations. The increase of the amount of valuable materials is one goal of the research projects described here. Opportunities for an improved separation of metals from small electrical devices were examined in the first treatment using commercially available and cost-effective processing technology and optimization potentials by using a developed multi-metal separator. Problems that may arise in this context and aspects of representative waste sampling of shredded electronic waste $<8 \mathrm{~mm}$ were considered, too.

Dr.-Ing. C. Borowski ( $\triangle$ )

Hochschule Nordhausen,

Helmestraße 94,

99734 Nordhausen, Deutschland

christian.borowski@hs-nordhausen.de
Keywords: Valuable materials recovery, Waste electrical equipment, Small electrical household appliances, Electronic scrap, First treatment, Magnet, Separation, Fine grain, Multi-metal separator, Sampling

\section{Forschungsumfeld}

Das Vorhaben mit dem Titel „Innovationsbasierte Steigerung der Wertstoffrückgewinnung" wurde von der Hochschule Nordhausen unter Mithilfe des Instituts für Angewandte Bauforschung Weimar gGmbH bearbeitet und durch den Freistaat Thüringen aus Mitteln des Europäischen Sozialfonds gefördert. Es adressierte mit der Optimierung der Abscheidung von Metallen und kritischen Rohstoffen aus Elektroaltgeräten (EAG) beim Erstbehandler ein wichtiges Forschungsfeld im Recyclingbereich. Das Projekt Multimetallseparator lief vom 01.01.2018 bis zum 31.12.2020 und hatte zum Ziel, Elektroschrott mit KorngröBen $<8 \mathrm{~mm}$ durch Verwendung abgestufter Magnetfelder aufzubereiten. Die Hochschule Nordhausen und die Firma B\&F Metallbautechnik GmbH danken dem Bundesministerium für Wirtschaft und Energie für die Finanzierung dieser Untersuchung im Rahmen des ZIM-Programms unter Förderkennzeichen ZF4079008KO8. Bearbeitet wurden die Vorhaben im Thüringer Innovationszentrum für Wertstoffe (ThIWert) in Nordhausen. Die Förderung des Aufbaus der Forschungseinrichtung erfolgt durch den Freistaat Thüringen und wird aus Mitteln des Europäischen Fonds für regionale Entwicklung kofinanziert.

\section{Separation von Metallen aus Elek- trokleingeräten mittels FE- und NE- Scheidung}

Hauptbestandteil von Elektroaltgeräten sind unterschiedliche Kunststoff- und Metallarten sowie kritische Rohstoffe. Um diese bestmöglich zurückzugewinnen und für die Wirt- 
schaft nutzbar zu machen, müssen die verschiedenen Wertstoffe aufgeschlossen und mit einem vertretbaren Aufwand separiert werden.

Hierzu erfolgt in einem ersten Behandlungsschritt eine Entnahme von Gefahrstoffen. Anschließend werden die Elektroaltgeräte in eine spezialisierte Aufbereitungsanlage geliefert oder direkt durch den optierten Erstbehandler einem qualifizierten Aufschluss unterzogen. In den Untersuchungen wurde überprüft, ob durch Verwendung von robusten, am Markt verfügbaren und vergleichsweise kostengünstigen Aufbereitungsaggregaten eine effektive Abscheidung von Wertstoffen bei kleinen optierten Erstbehandlungsanlagen durchführbar ist. Im Fokus standen hier Elektrokleingeräte der Sammelgruppe 5 (SG5), die bei direktem Verkauf derzeit kaum Erlöse erzielen.

Die für die Versuche verwendeten Elektrokleingeräte der SG5 (siehe beispielhafte Abb. 1) stammten von der Nordthüringer Werkstätten gGmbH, einem optierten Entsorgungsfachbetrieb für Elektroaltgeräte sowie Einrichtung für Menschen mit Behinderung. Gleichzeitig übernimmt die Einrichtung die Sammlung von Elektroaltgeräten im Landkreis Nordhausen.

Mit Hilfe eines Einwellenzerkleinerers der Firma Weima wurden die repräsentativ zusammengestellten Elektrogeräte auf eine Korngröße von $\leq 30 \mathrm{~mm}$ zerkleinert und in einen Aufgabebunker gefördert. Anschließend wurde das Material mittels Schwingförderer gleichmäßig auf ein Förderband aufgegeben. Mittels Überbandmagnet fand die Abscheidung einer ersten magnetischen Fraktion statt. Das Durchgangsmaterial wurde weiter auf eine Magnettrommel mit eingebautem Starkfeldmagneten transportiert, der als Schutz für die Poltrommel sowie das Förderband des Wirbelstromscheiders fungiert und schwach magnetische Materialien aus dem Input entfernen soll. Das nicht magnetisierbare Material wurde im Anschluss über eine Vibrationsförderrinne auf dem Beschleunigungsband des NE-Abscheiders verteilt. Durch die Induktion von Wirbelströmen wurden Nichteisenmetalle über einen

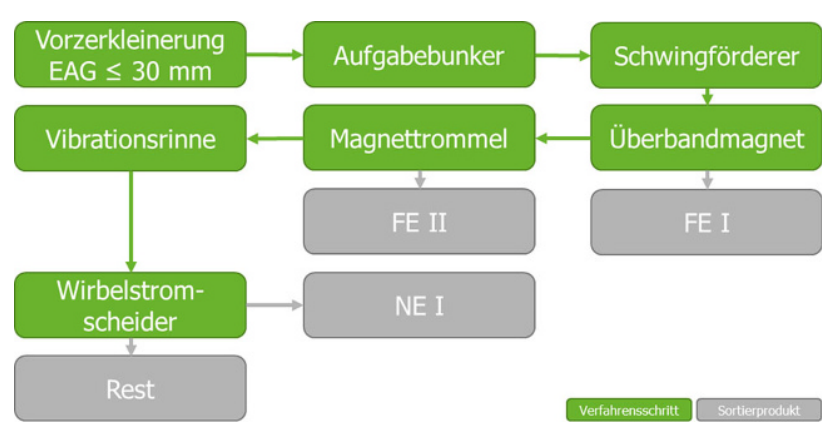

Abb. 2: Verfahrensablaufzur Abscheidung von FE- und NE-Metallfraktionen SG5

Trennscheitel ausgelenkt und so vom Restmaterial abgetrennt. Eine Darstellung des Sortierprozesses wird in Abb. 2 dargestellt.

Durch die gerade beschriebenen Separationsprozesse konnten zwei Eisenfraktionen, eine Nichteisenfraktion sowie eine Restfraktion erzeugt werden. Diese Fraktionen wurden anschließend ausgewogen und mit dem Eingangsmaterial ins Verhältnis gesetzt. Auf Basis der Berechnungen konnte ermittelt werden, dass in der Fraktion Eisen I 18,7 M.$\%$, in der Fraktion Eisen II 8,9 M.-\% und in der Fraktion Nichteisenmetalle I 6,9 M.-\% des Ausgangsmaterials abgeschieden wird. Das Durchgangsmaterial entsprach damit einem Anteil von 65,4M.-\% und bestand augenscheinlich zum größten Teil aus Glas, Kunststoffen und anderen organischen Fraktionen.

Um Aussagen über eine Vermarktbarkeit und Ausbringung von Wertstoffen der erzeugten Fraktionen zu ermöglichen, wurden diese einer händischen Analyse unterzogen. Eine Unterteilung wurde in Kupferkabel, Platinen und Zielfraktion gewählt, um exakte Werte für den Anteil des Fehlaustragsmaterials sowie die Gründe für den Austrag feststellen zu können. Die Bestimmung der einzelnen Masseanteile erfolgte je Austragsfraktion. Hierbei wurden die Einzelmassen der händisch generierten Sortiergruppen durch

Abb. 1: Exemplarische Fraktion Elektrokleingeräte SG5

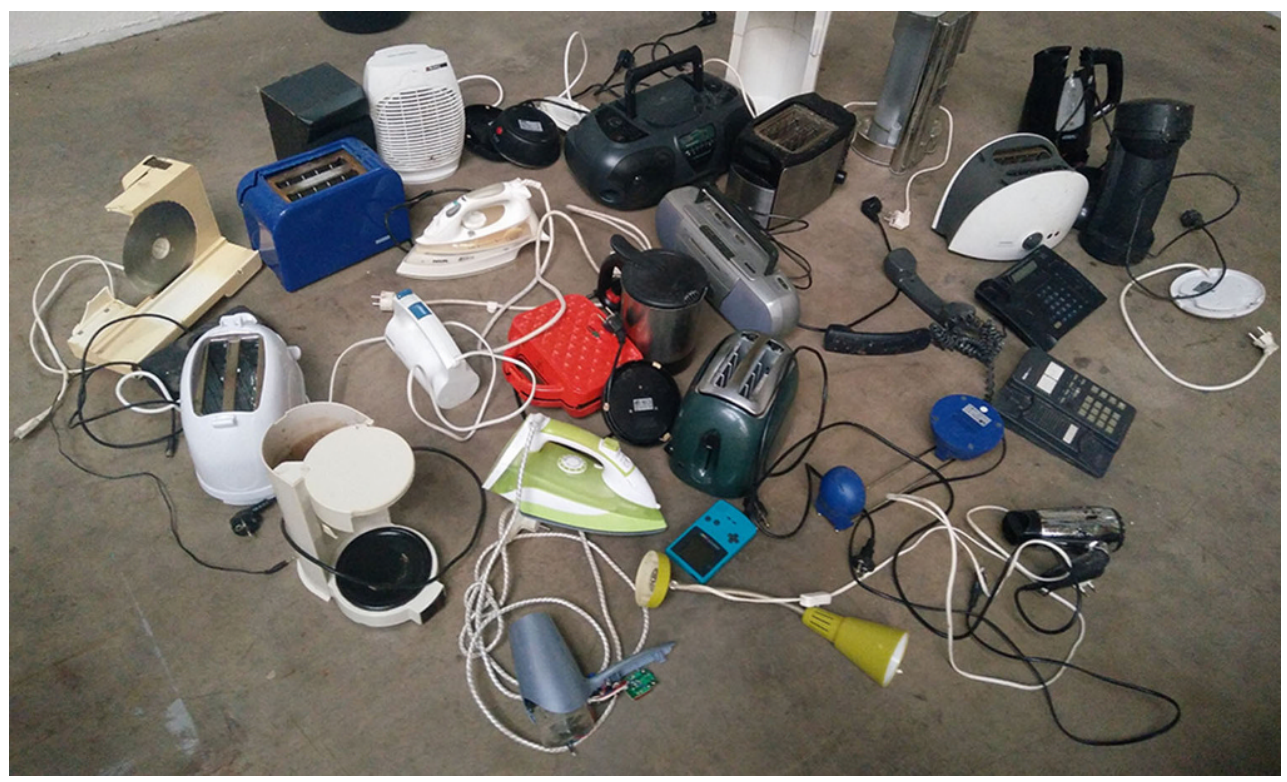


Abb. 3: Zusammensetzung abgeschiedene Zielfraktionen

Abb. 4: Potenzielle Erlöse bei vollständigem Ausbringen werthaltige Metallfraktionen
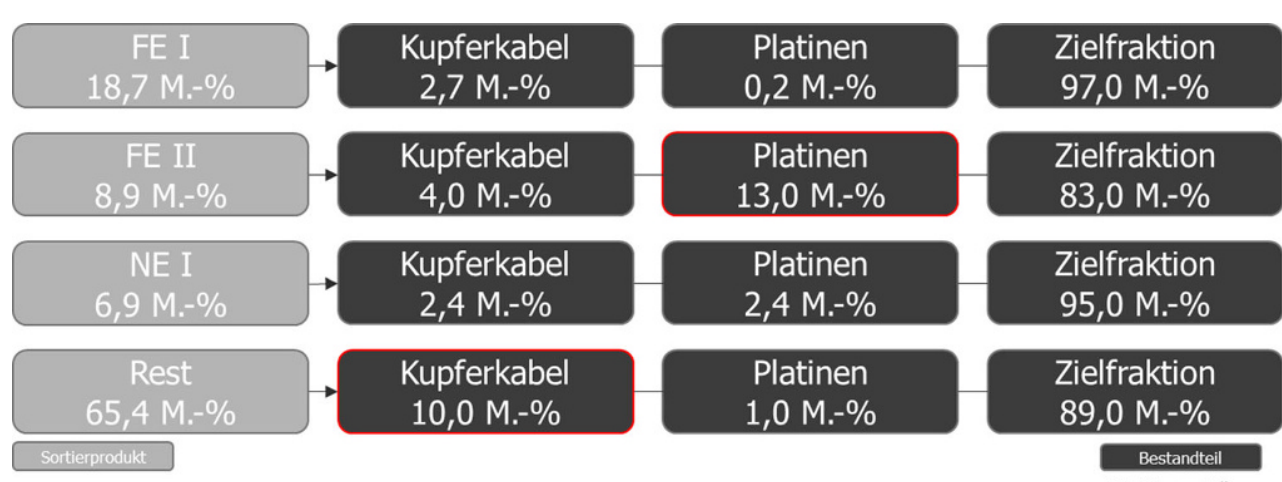

Zielfraktion 95,0 M.-\%

Zielfraktion 89,0 M. $\%$

Kupferkabe 10,0 M. $-\%$
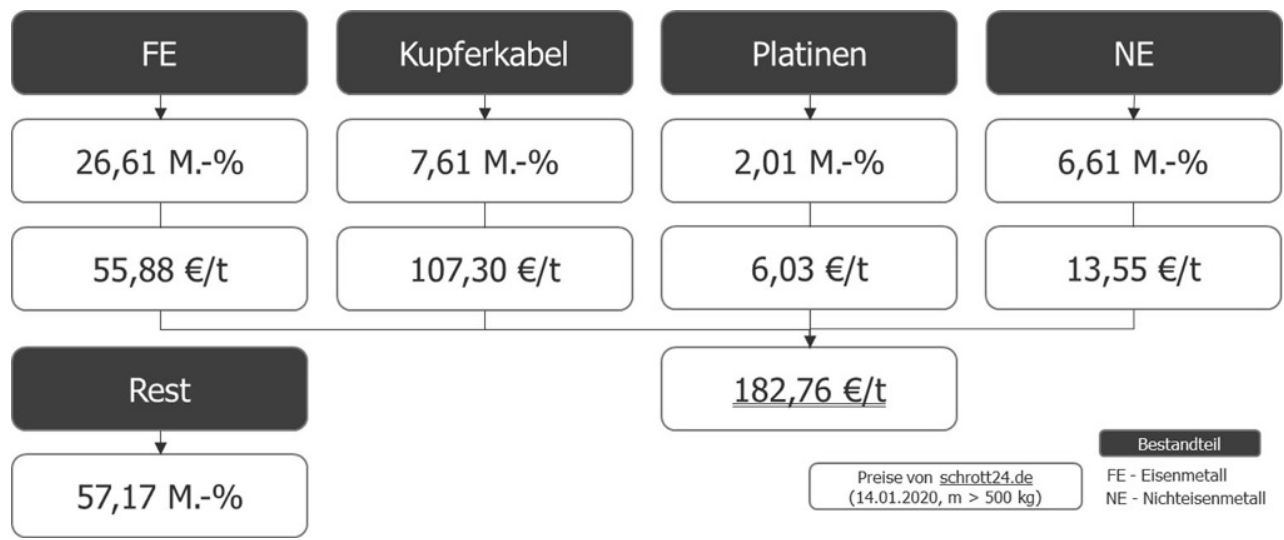

Bestandteil

FE- Eisenmetall

NE - Nichteisenmetall

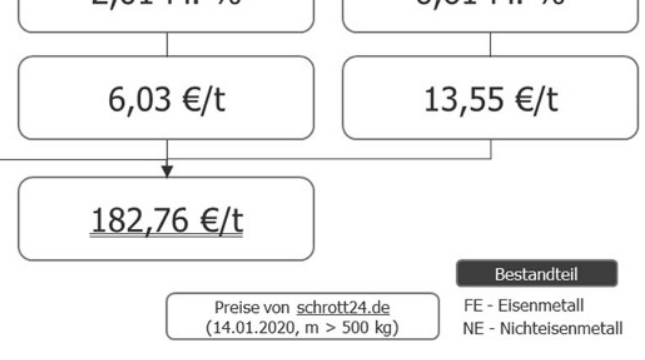

die Gesamtmassen der einzelnen Fraktionen FE I, FE II, NE I und Rest geteilt (Abb. 3).

Im Ergebnis kam es in beiden Eisenfraktionen sowie in der Restfraktion zur Verschleppung von Kupfer. Während beim Trennschritt FE I verschraubte Kontakte mit anhaftenden Kabeln für einen Austrag von Kupferkabeln sorgten, waren es im Bereich der FE II Fraktion hauptsächlich Platinen, die für eine Verunreinigung der Zielfraktion verantwortlich sind.

In Geräten der SG5 werden aus Kostengründen vermehrt Low-Grade Leiterplatten (Leiterplatten Klasse 3) verwendet. Diese Platinen sind mit großen Bauteilen bestückt. Dabei handelt es sich häufig um Tantal-Kondensatoren, die nur sehr schwer magnetisierbar sind und dadurch erst am Starkfeldmagneten abgeschieden werden können. Da der Aufschluss der Elektrokleingeräte bei $\leq 30 \mathrm{~mm}$ die Platinen nicht vollständig entstückt, sorgen die schwer magnetisierbaren Bestandteile für eine Ausschleusung der Leiterplattenstücke in die Fraktion FE II. Gleichzeitig können die vorhandenen kleinen Schrauben den gleichen Effekt bewirken.

Der Anteil an Kupferkabeln in der Restfraktion ist nach der händischen Sortierung hoch. Aufgrund ihrer länglichen Form können bei der Wirbelstromscheidung keine ausreichenden Wirbelströme in die zum größten Teil noch ummantelten Kabel induziert werden. Somit erfolgt kein Austrag.

Im Projekt sollten ebenfalls potenzielle Wertgehalte der verschiedenen metallhaltigen Fraktionen ermittelt werden. Hierzu wurden die Ergebnisse der händischen Sortierung der zerkleinerten Elektroaltgeräte der SG5 genutzt und diese mit Marktpreisen multipliziert. Die erhaltenen Ergebnisse sind in Abb. 4 dargestellt und beziehen sich jeweils auf eine Tonne des Materials. Bei einer theoretisch vollständigen Ausbringung der unterschiedlichen Metalle lassen sich $182,76 €$ pro Tonne Elektroaltgeräte der SG5 erlösen. Es ist jedoch zu beachten, dass die Restfraktion, sofern keine weitere Aufbereitung erfolgt oder ein Abnehmer vorhanden ist, kostenpflichtig entsorgt werden muss.

Die Versuche haben gezeigt, dass der Einsatz von FE- und NE-Scheidern nur bedingt für den Einsatz beim Erstbehandler geeignet ist. Für eine frühzeitige Entschlackung des Sortierprozesses durch Abscheidung von magnetischen Materialien erscheint die Verwendung eines Überbandmagnetes lohnend, da sich die Verschleppung von Kupfer und anderen Materialien in den durchgeführten Versuchen in engen Grenzen hielt. Hierzu muss jedoch der Abstand des Magnetes über dem Transportband sowie die durch die Anlage transportierte Schichtdicke des Inputmaterials optimal eingestellt und reguliert werden, um die Ausleitung von kupferhaltigen Materialien bestmöglich zu unterbinden.

Zum Schutz des Wirbelstromscheiders ist es unabdingbar, schwach magnetische Bestandteile aus dem Prozess abzuscheiden. Daher wird es im Austrag der Magnettrommel immer zu Verschleppungen von Platinen kommen.

Durch die stark heterogene Zusammensetzung der metallhaltigen Bestandteile der SG5 lässt sich eine gezielte Ausleitung von einzelnen NE-Metallen nicht gewährleisten. Es wird ein Gemisch von unterschiedlichen NE-Metallen erzeugt, die in dieser Form sonst hauptsächlich bei der 
Abb. 5: Verschlepptes Wertstoffpotential in Restfraktion
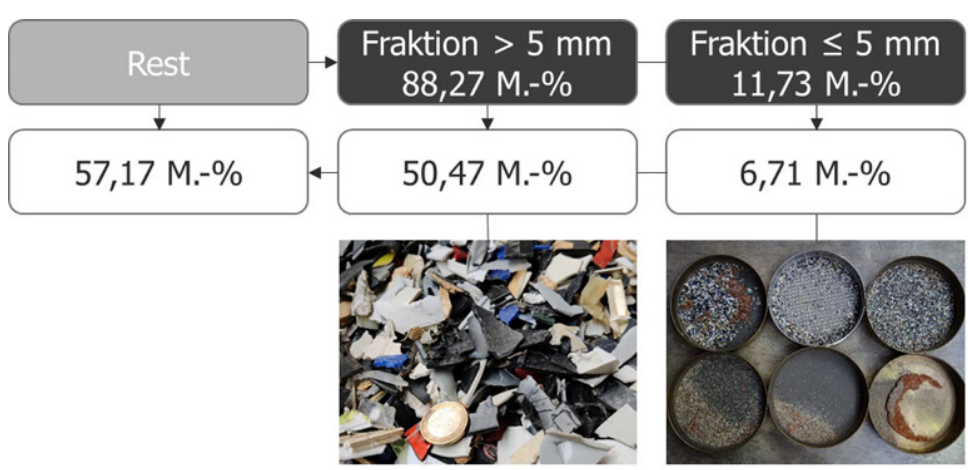

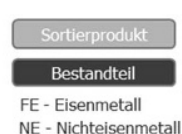

MVA-Schlackenaufbereitung entstehen. Gleichzeitig reichen die Querschnitte der noch ummantelten Kupferkabel nicht aus, einen ausreichend hohen Wirbelstrom zu induzieren, um diese auszuleiten. Sie gelangen daher in das Durchgangsmaterial, also den kunststoffhaltigen Rest des Aufbereitungsprozesses.

Als Alternative zur NE-Scheidung können sensorbasierte Trennsysteme angesehen werden. Erste eigene Versuche mit diesen Systemen haben aufgezeigt, dass eine Abtrennung der bereits beschriebenen Zielfraktionen Eisen, Nichteisen, Kupferkabel und Platinen möglich ist. Durch Kombination von optischen sowie induktiven Sensoren in Form eines Bandsortierers auf Druckluftbasis kann hier ein zielgerichteter Austrag realisiert werden. Eine Sortierung der Wertstoffe kann durch eine Kreislaufführung des Inputmaterials in einzelnen Batchdurchgängen erfolgen. Gleichzeitig kann die Restfraktion, die hauptsächlich aus Kunststoffen besteht, durch Nutzung von NIR-Technik ebenfalls in die einzelnen Bestandteile getrennt werden. Diesbezüglich sind zukünftig noch weitere Versuche im ThIWert geplant.

Aufgrund der Anschaffungskosten eines solchen Sortierers ist die Rentabilität für einen einzelnen Erstbehandler sehr stark von durchschnittlichen Input-Jahresmengen in der SG5 sowie Rohstoffpreisen abhängig und derzeit nicht gegeben.

\section{Problematik der Feinkornbildung bei Zerkleinerung von Elektrokleingeräten}

Die Restfraktion weist neben den bereits angesprochenen Bestandteilen, wie Kunststoffe, weitere organische Materialien und Glas, ebenfalls einen Feinanteil auf, der bei den hier beschriebenen Versuchen bei einem Siebschnitt $\leq 5 \mathrm{~mm} 6,71 \mathrm{M}$.- $\%$ des Inputmaterials ausmacht. Dieser Anteil ist stark abhängig vom gewählten Zerkleinerungsaggregat, der Beschaffenheit der Ausgangsmaterialien sowie der gewünschten Zielkorngröße.

In diesem Korngrößenbereich von $\leq 5 \mathrm{~mm}$ sammeln sich außerdem magnetisierbare Bestandteile aus Bauteilen von Elektrokleingeräten wie Lautsprechern oder Kondensatoren an, die mit verschiedenen Nichteisenmetallen Agglomerate bilden. Dies kann bei einem gesteigerten Anteil an Multimediageräten mit geringen Leiterquerschnitten der Kabel ebenfalls zu den bereits angesprochenen Fehlausträ- gen (Abb. 5) führen und so nachfolgende Aufbereitungsschritte stören. Grund hierfür ist, dass die magnetisierten Agglomerate zerkleinerte Kabellitzen umschließen können.

Diese Fraktion soll mit Hilfe des Multimetallseparators über eine gezielte Abscheidung der magnetisierten Bestandteile in eine hochwertige Fraktion für statische Separatoren oder die Nasstrenntische aufbereitet werden.

\section{Einsatz eines Multimetallseparators}

In den Recyclingbetrieben werden nach der Zerkleinerung des Schrotts u.a. Sortiergeräte eingesetzt, die die Materialien hinreichend genau detektieren und ausschleusen. Dies funktioniert für Korngrößen $>20 \mathrm{~mm}$ gut. Jedoch können kleine Körner nur mit erhöhtem Aufwand erfasst und von speziell auf diesen Korngrößenbereich angepassten Maschinen, wie bspw. Nasstrenntischen, getrennt werden. Im Zerkleinerungsprozess entstehen Fraktionen mit einer Korngröße $<8 \mathrm{~mm}$, die über 50 M.- \% magnetisches und magnetisierbares Material enthalten. Der hohe magnetische Anteil kann dazu führen, dass Maschinenteile verstopfen und die Sortierleistung sinkt. Zur Entlastung dieser Apparate und zur Ausschleußung/Herstellung hochwertiger, magnetisierbarer Produkte wird der Multimetallseparator entwickelt.

Der Einsatz von Magnetabscheidern ist in der Industrie ein etabliertes Verfahren. Hierbei werden i. d. R. Elektromagneten verwendet. Im Bereich der Qualitätssicherung von Lebensmitteln finden Kassettenmagnete Anwendung, die über Permanentmagnete verfügen und direkt in den Stoffstrom eingebracht werden. Diese beiden Abscheidetechniken haben gemeinsam, dass sie nicht komplex aufgebaut sind und ein konstantes Magnetfeld über die Fläche ausbilden. Eine selektive Anpassung der Magnetfeldstärke ist nicht möglich. Dies führt nicht nur zu einem hohen Strom-/ Magnetstabverbrauch, sondern kann auch den Abscheidegrad negativ beeinflussen. Im Projekt „Multimetallseparator" ist u. a. ein Ziel, auf die zu separierenden Produkte abgestimmte Magnetfelder einzusetzen. Wie in Abb. 6 dargestellt, soll ein magnetisches und ein nichtmagnetisches Produkt erzeugt werden.

Dabei kann das magnetische Produkt 1 in weitere Produkte getrennt werden, je nach angewandter Feldstärke (Abb. 7). Somit wird die unterschiedliche Magnetisierungs- 


\begin{tabular}{|c|c|}
\hline \multicolumn{2}{|c|}{$\begin{array}{c}\text { Elektroschrott zerkleinert }<8 \mathrm{~mm} \\
100 \% \\
\mathrm{~m}_{\text {in }}\end{array}$} \\
\hline \multicolumn{2}{|c|}{ Multimetallseparator } \\
\hline \begin{tabular}{|c|} 
Produkt 1 \\
magnetisch \\
z. B. 30\% \\
m $_{\text {mag }}$
\end{tabular} & $\begin{array}{c}\text { Produkt 2 } \\
\text { nicht magnetisch } \\
\text { z. B. } 70 \%\end{array}$ \\
\hline
\end{tabular}

Abb. 6: Funktionsprinzip Multimetallseparator

fähigkeit von Materialien ausgenutzt. Die Entnahme erfolgt über gestufte Magnetfelder, beispielhafte Ergebnisse sind in Abb. 8 dargestellt.

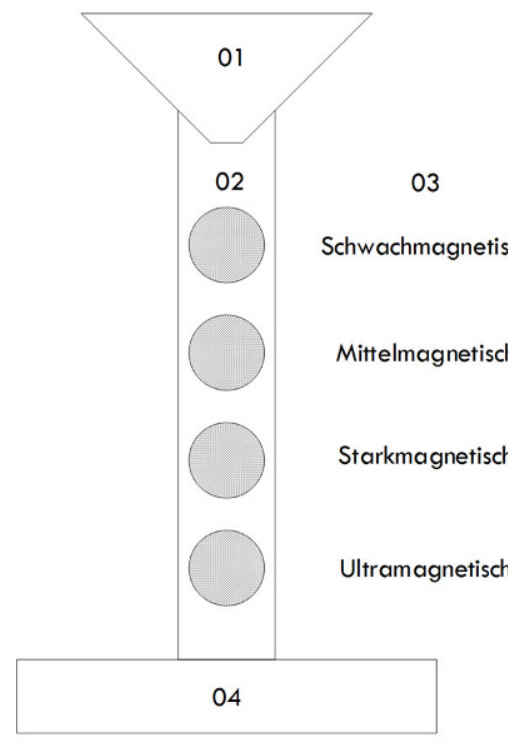

a

Abb. 7: Schema Multimetallseparator und Multimetallseparator-Fallturm
Vier verschiedene Einsatzbereiche in einer Elektronikschrottaufbereitung sind für den Einsatz des Multimetallseparators vorgesehen:

- nach dem Schredder,

- zur Kupferanreicherung

- für die Tantalanreicherung aus zerkleinerten Elektroaltgeräten und

- zum Einsatz im Bereich des Li-Ionen-Batterierrecyclings.

\section{Probenahme und Analytik für E-Schrott mit Hilfe des Probenahmebettes - Erste Ergebnisse in Vorbereitung auf die Normung}

Probleme bei der Durchführung von Sortieranalysen von zerkleinerten Elektroaltgeräten bereiten fehlende Möglichkeiten zur repräsentativen Reduzierung der zu beproben-

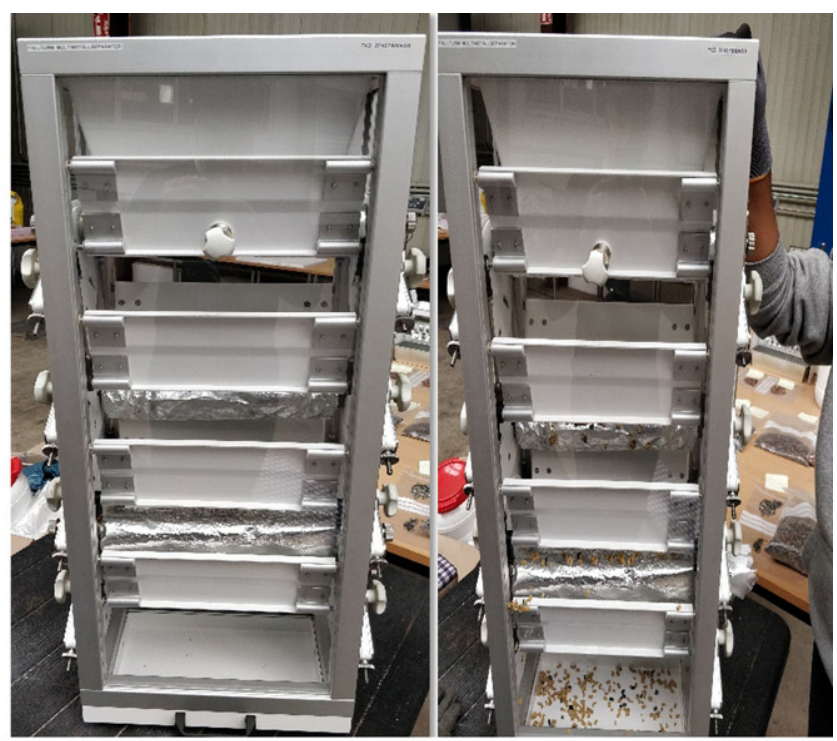

b

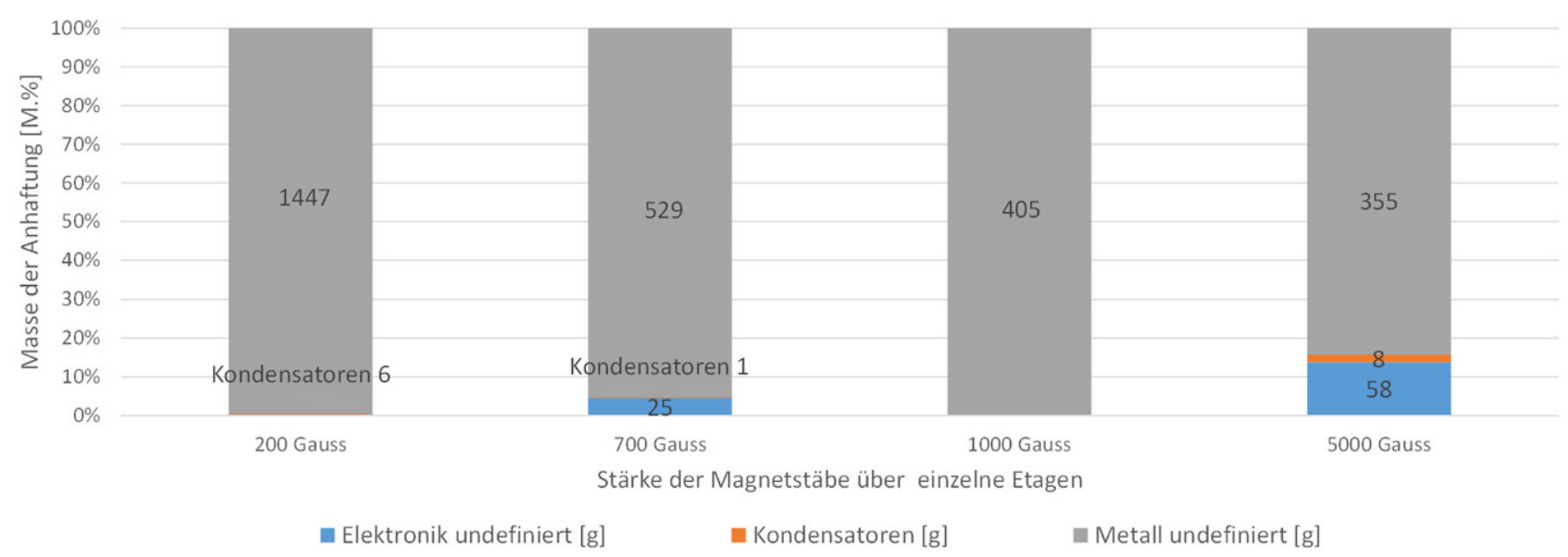

Abb. 8: Anhaftung magnetisches Material an Magnetstäben 
Abb. 9: Anknüpfungspunkt Probenahme

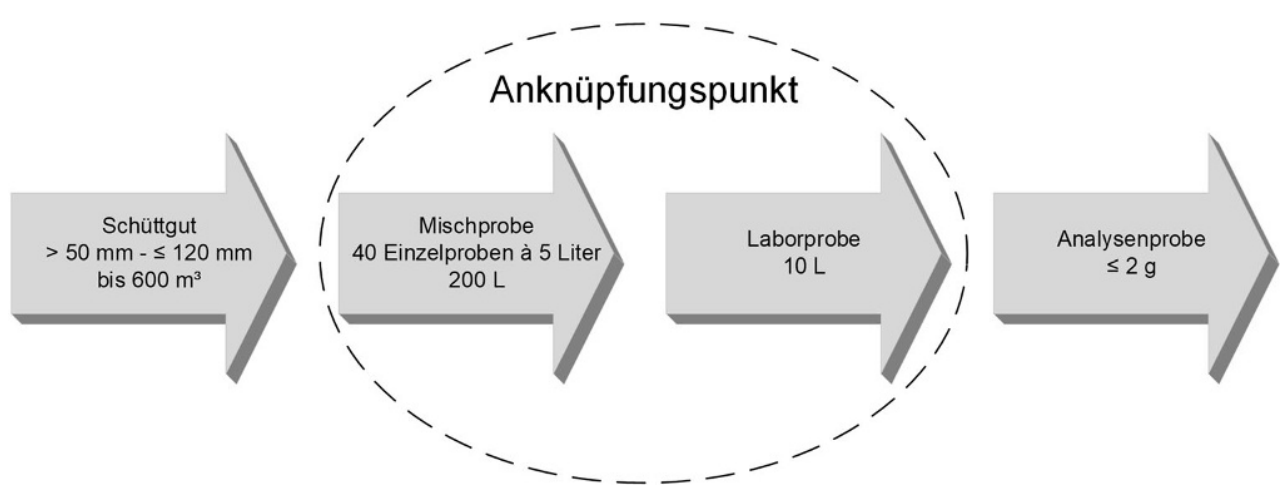

Nicht repräsentativ beprobungsfähig

Repräsentativ beprobungsfähig
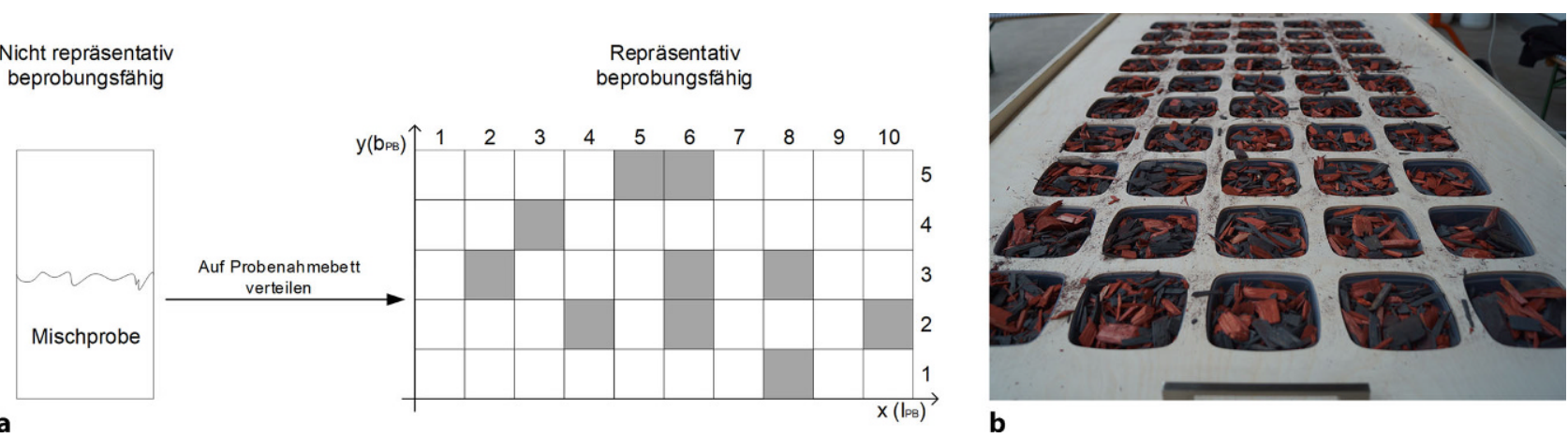

Abb. 10: Probenahmebett und Befüllung mit Rindenmulch

den Fraktionen über 10I Probevolumen (Abb. 9). Auf Basis der bereits erlangten Erkenntnisse im Bereich der Probenahme an der Hochschule Nordhausen wurde eine neue Methode zur Probenverjüngung entwickelt.

Bei der Verwendung eines sogenannten Probenahmebettes (Abb. 10) wird ein Probevolumen auf ein Raster gegeben. Hier befinden sich 50 gleichmäßig verteilte Behälter mit einem einheitlichen Volumen von einem Liter. Aufgeteilt ist das Probenahmebett in fünf Behälterreihen in y-Achse und zehn Reihen in x-Achse. Das Probematerial, welches schütt- sowie rieselfähig ist und eine Korngröße von $<40 \mathrm{~mm}$ besitzt, wird auf das "Bett" aufgegeben und damit in die in die Oberfläche eingelassenen Behälter gefüllt.
Anschließend wird unter Zuhilfenahme eines Zufallsgenerators und eines Rasters eine vorgegebene Anzahl Behälter entnommen, die in Summe einer Laborprobe von zehn Litern entsprechen.

Zur Analyse wurde die Methode des Bestimmtheitsmaßes angewendet. Je geringer die Masseabweichung unter den Probebehältern, umso mehr strebt das Ergebnis des Bestimmtheitsmaßes gegen null. Im ersten Versuch wurde handelsüblicher Rindenmulch (Korngröße 0 bis $135 \mathrm{~mm}$ ) drei verschiedener Farben volumengleich zu einer 50 I Gesamtprobe vermischt, anschließend auf das Probenahmebett gegeben und danach zehn Probebehälter mit einem Liter Volumen zufällig entnommen. Alle Partikel wurden ein-
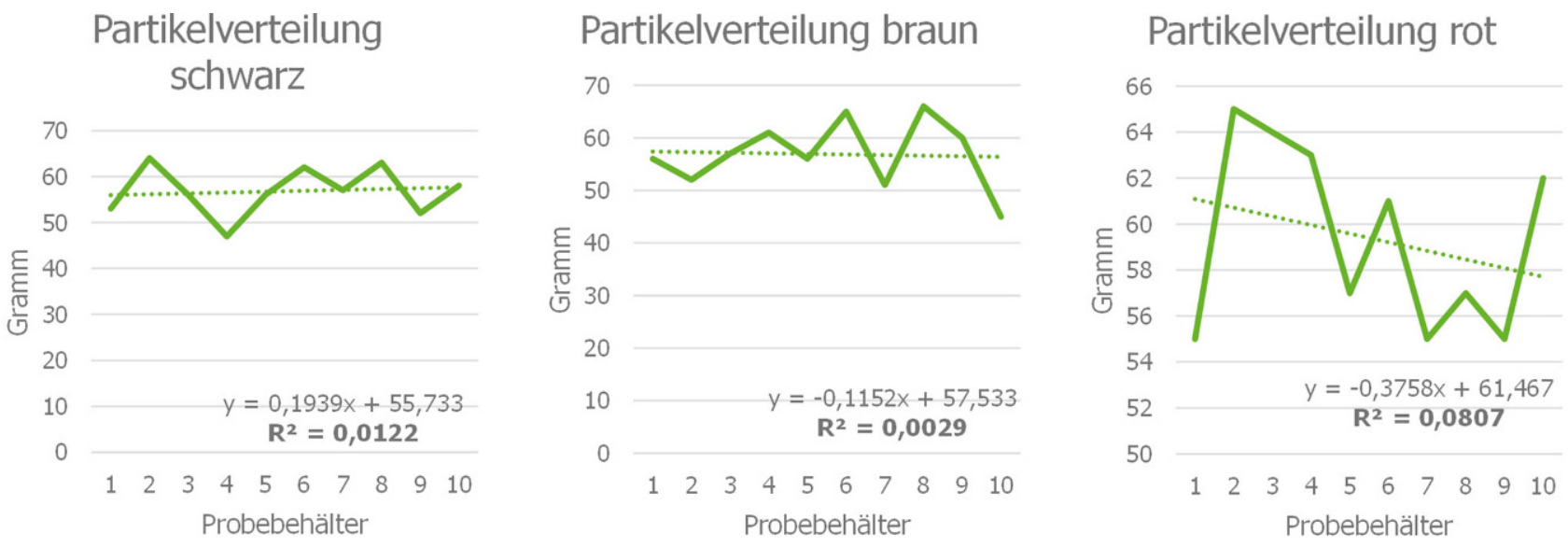

Abb. 11: Analyseergebnisse Partikelverteilung Rindenmulch 

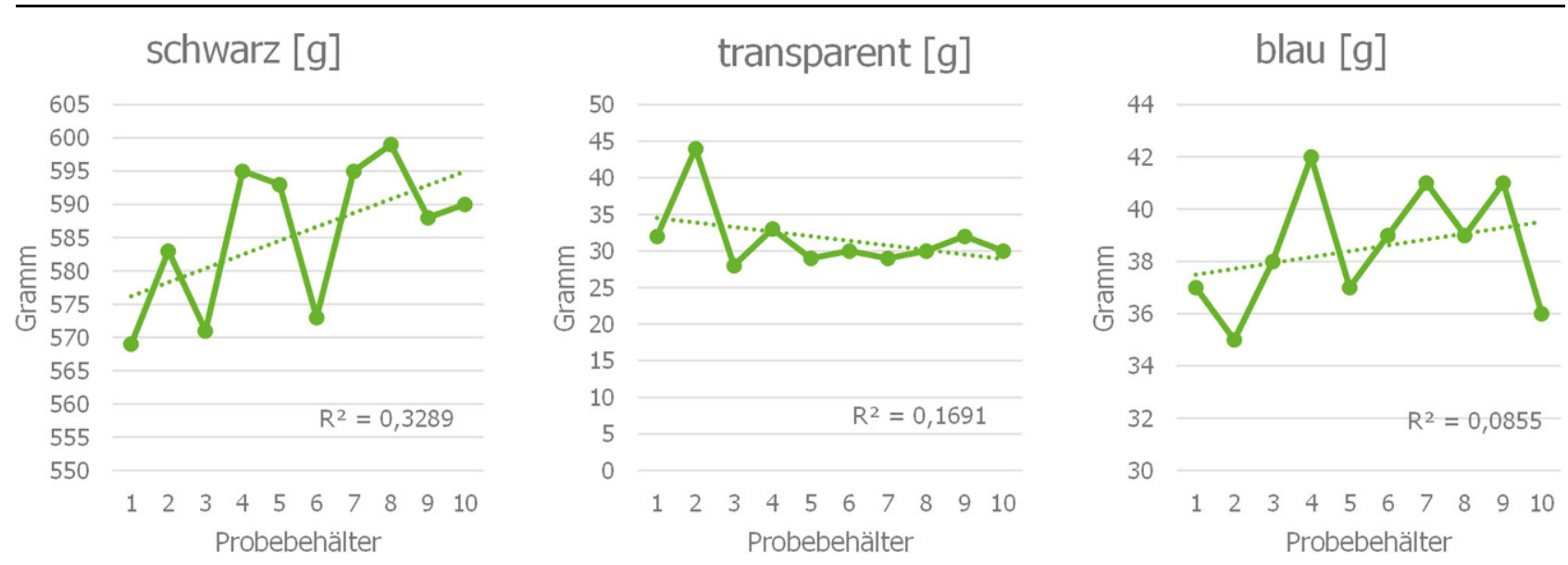

Abb. 12: Analyseergebnisse Partikelverteilung Kunststoffgranulat

zeln händisch aus den ein Liter Behältern sortiert und anschließend ausgewogen. Das Bestimmtheitsmaß (Abb. 11) liegt bei den Farben Schwarz, Braun und Rot zwischen 0,12 und 0,081 .

Im zweiten Versuch wurde eine bereits vorhandene Kunststoffgranulatmischung (Korngröße 2,9 bis 5,1 mm) aus den Farben Schwarz, Transparent und Blau verwendet. Die Beprobung fand nach dem Prinzip des Rindenmulchs statt. Das Bestimmtheitsmaß (Abb. 12) für die Farben Schwarz, Transparent und Blau liegt zwischen 0,33 und 0,086 . Somit ist die Varianz bei dem Material Kunststoffgranulat höher.

Die Probeneigenschaft Masse in den jeweils 10 zufällig entnommenen Probebehältern schwankt tolerabel und kann somit auf Elektroschrott mit Korngrößen kleiner $8 \mathrm{~mm}$ übertragen werden.

Danksagung. Das Projekt Multimetallseparator lief vom 01.01.2018 bis zum 31.12.2020. Die Hochschule Nordhausen und die Firma B\&F Metallbautechnik $\mathrm{GmbH}$ (Wildeshausen) danken dem Bundesministerium für Wirtschaft und Energie für die Finanzierung dieser Untersuchung im Rahmen des ZIM-Programms unter Förderkennzeichen ZF4079008KO8. Wir danken weiterhin dem Freistaat Thüringen, welcher die Forschungsarbeiten aus Mitteln des Europäischen Sozialfonds unterstützt hat.

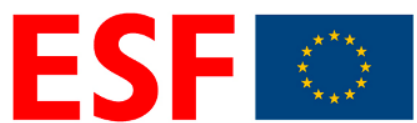

EUROPA FÜR THÜRINGEN EUROPÄISCHER SOZIALFONDS

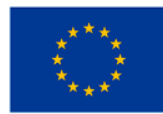

EUROPÄISCHE UNION

Europäischer Sozialfonds

Gefördert durch:

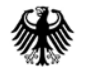

Bundesministerium

für Wirtschaft

und Energie

aufgrund eines Beschlusses des Deutschen Bundestages

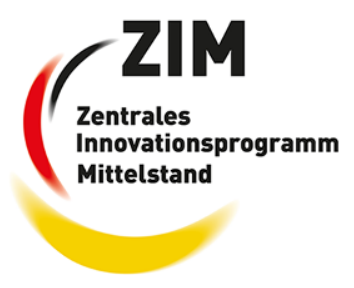

Funding. Open Access funding enabled and organized by Projekt DEAL.

Open Access Dieser Artikel wird unter der Creative Commons Namensnennung 4.0 International Lizenz veröffentlicht, welche die Nutzung, Vervielfältigung, Bearbeitung, Verbreitung und Wiedergabe in jeglichem Medium und Format erlaubt, sofern Sie den/die ursprünglichen Au- 
tor(en) und die Quelle ordnungsgemäß nennen, einen Link zur Creative Commons Lizenz beifügen und angeben, ob Änderungen vorgenommen wurden.

Die in diesem Artikel enthaltenen Bilder und sonstiges Drittmaterial unterliegen ebenfalls der genannten Creative Commons Lizenz, sofern sich aus der Abbildungslegende nichts anderes ergibt. Sofern das betreffende Material nicht unter der genannten Creative Commons Lizenz steht und die betreffende Handlung nicht nach gesetzlichen Vorschriften erlaubt ist, ist für die oben aufgeführten Weiterverwendungen des Materials die Einwilligung des jeweiligen Rechteinhabers einzuholen.

Weitere Details zur Lizenz entnehmen Sie bitte der Lizenzinformation auf http://creativecommons.org/licenses/by/4.0/deed.de.

Hinweis des Verlags. Der Verlag bleibt in Hinblick auf geografische Zuordnungen und Gebietsbezeichnungen in veröffentlichten Karten und Institutsadressen neutral. 